\title{
A model-driven approach for engineering customizable MES with the application to the food and beverage industry
}

\author{
Xinyu Chen ${ }^{1}$ (D) $\cdot$ Christoph Nophut $^{1} \cdot$ Tobias Voigt $^{1}$ \\ Received: 16 December 2020 / Accepted: 20 May 2021 / Published online: 27 May 2021 \\ (C) The Author(s) 2021
}

\begin{abstract}
The Manufacturing Execution System (MES) is a process-oriented IT solution collecting and managing information from the shop floor manufacturing processes. Because of the programming and customization effort required for specific production processes, the MES implementation is not widespread in the food and beverage industry, as most food and beverage manufacturers are small- and medium-sized enterprises with limited resources to invest in MES. For engineering the MES that should be customized according to the target production processes, a model-driven approach has been presented in this paper with six phases covering the entire lifecycle of the MES engineering process. By using this approach, MES can be automatically generated and sustainably improved, which has the potential to reduce the complexity of implementation as well as the resources required for the engineering of customizable MES. Based on two use cases in the processing and packaging areas in the food and beverage industry, the feasibility and practicality of the presented approach have been proven.
\end{abstract}

Keywords Manufacturing Execution Systems · Model-driven engineering; MES modeling · Food and beverage industry

\section{Introduction}

The Manufacturing Execution System (MES) is a processoriented software system for managing and analyzing information based on real-time data accrued from manufacturing processes. It connects the automation and enterprise layers in industrial manufacturing architecture. On one hand, MES guides the implementation of rough production plans from enterprise systems, such as the Enterprise Resource Planning (ERP) systems, into detailed operations for technical systems on the shop floor. On the other hand, it provides the enterprise with production key performance indicators (KPIs), such as energy consumption, saleable output, and machine efficiency, which assist commercial decisions to be taken and improve the performance of production processes. The Manufacturing Enterprise Solution Association (MESA) has proposed a formal definition describing the essential role of the MES in a manufacturing enterprise: "The MES delivers information that

Xinyu Chen

xinyu.chen@outlook.de

1 Chair of Food Packaging Technology, Technical University of Munich, Weihenstephaner Steig 22, 85354 Freising Munich, Germany enables the optimization of production activities from order launch to finished goods. Using current and accurate data, the MES guides, initiates, responds to, and reports on plant activities as they occur. The resulting rapid response to changing conditions, coupled with a focus on reducing non-value-added activities, drives effective plant operations and processes. The MES improves the return on operational assets as well as ontime delivery, inventory turns, gross margin, and cash flow performance. The MES provides mission-critical information about production activities across the enterprise and supply chain via bi-directional communications" [1]. To cover all activities in the production environment, a series of MES functions have been defined in [1-3], such as production tracking, fine scheduling, performance analysis, and energy management. Implementing the MES can improve the transparency of manufacturing processes so that the quality of products and efficiency of workflow can be optimized [4].

\subsection{The Food and beverage industry and MES}

The largest manufacturing sector in the European Union (EU) is the food and beverage sector, with a direct turnover of $€ 1192$ billion and more than 4.7 million employees in 2019 [5]; it is also the largest energy consumer compared to other sectors [6]. Food and beverage manufacturing enterprises are 
under pressure to control production costs and provide products at reasonable prices in the competitive market. Due to rising energy prices [7], new environmental regulations with associated costs for $\mathrm{CO}_{2}$ emissions [8,9], and the growth of awareness of environmental issues from customers [10], the improvement of energy efficiency is therefore necessary for the manufacturers in the food and beverage industry. To illuminate the relationship between environmental and economic benefits and further evaluate the sustainability of manufacturing processes, eco-efficiency indicators (EEIs) for the food and beverage industry were developed, in which process parameters and shop floor production data must be taken into consideration [11]. Since the MES is connected to the shop floor and can directly monitor and control the production processes, it supports production managers and process owners in better (i) understanding energy consumption in the process, (ii) calculating their own energy indicators, and (iii) exploring energy saving potentials. The ability of the MES managing and reducing energy consumption in the manufacturing process has been indicated in [12].

The food processing industry needs efficiency-improving production methods to reduce production cost and to comply with increased regulations [13]. Weinekotter [14] indicated that the food packaging machinery remains severely underutilized, which can be attributed to shorter production runs and frequent changeovers. For companies to be production efficient, the development of more efficient production techniques to increase the overall equipment effectiveness (OEE) is required. Lean manufacturing is considered as a potential methodology to improve productivity and further decrease production cost in manufacturing organizations. Research by Borges Lopes et al. [15] indicated that improved productivity and production flexibility in food and beverage manufacturing could be realized by applying lean manufacturing principles and tools. Based on the collection and analysis of production data, Desai et al. [16] applied the Six Sigma methodology on a pilot milk powder packaging line, which reduced the rejection rate of the final products and improved the annual financial benefits. The application of lean principles can be supported by implementing the MES, as useful real-time information can be provided by the MES to initiate, feed, or validate the lean decision-making processes to improve the efficiency [17]. Integrating the single minute exchange of die (SMED) principle into MES has been established to minimize time for machine changeover and enhance planning and production efficiency [18].

Vogel-Heuser et al. [19] proposed the automation diabolo as a global information architecture for industrial automation. MES, the essential information processing and delivery layer between business processes and control systems, is inseparable from modern technologies. In 2013, to enhance its country's position in global manufacturing, the German Government proposed Industry 4.0. Under this concept, the entire factory environment would become connected and enable mass customization through the application of advanced information and communication technologies in manufacturing [20]. The utility of the MES to the developing manufacturing industry was confirmed in [21] by analyzing the technologies that will accompany Industry 4.0. Along with the technology development, a platform- and interface-independent MES combined using service-oriented architecture and cloud computing can be achieved [22, 23].

According to a survey in the brewing industry regarding the implementation of MES [24], from the viewpoint of the system provider, the main benefits that MES can bring to manufacturers is the improvement of product tracking, increased production efficiency, the optimization of quality control, and efficient energy management. From the viewpoint of end-users, they indicated that their demands on MES are the increase in production efficiency, the improvement of product tracking, efficient energy management, and the optimization of machine maintenance.

\subsection{Model-driven engineering of the MES}

Although manufacturers in the food and beverage industry can be supported by the MES to optimize their processes and gain more transparency, and further improve the energy use and production efficiency. However, small- and medium-sized enterprises (SMEs), which consist the majority of the food and beverage industry (of the 285,000 companies in the EU, more than $99 \%$ are deemed SMEs [5]), are in an awkward position. Due to specific characteristics of the production process, lowprofit margins, and slow adaption of modern technologies, SMEs in the food and beverage industry have restricted resources invested in the conventional MES projects [25]. For each specific manufacturing process, the MES should be customized since it connected directly to the shop floor [26]. Consequently, the engineering costs of MES primarily arise from the programming effort for the customization, for example, the functionality of MES and the plants must be correspondingly adapted, and the interfaces of MES and programmable logic controllers (PLC) must be established [27, 28]. Instead of MES, many SMEs still use cheap but error-prone IT systems to provide some of the functionality of MES, such as KPI calculation and detailed production scheduling with spreadsheet programs [28]. For both sides involved in an MES project, namely the MES provider and manufacturer, a method with low programming and customizing efforts for the engineering of MES should be established. The use of the model-driven concept discussed here could be a solution.

Models are created to serve particular purposes, such as presenting a comprehensible description of some aspect of a system [29]. Model-driven engineering is an approach to reduce the conceptual gap between problem domains and software engineering [30]. The heterogeneity of the developed 
system and its concerns can be reflected by using models. In the model-driven engineering version of software development, models serve as the primary artifacts. The developers rely on computer-based technologies to transform these into running systems, which increase the reusability and reduce programming effort during the engineering process $[31,32]$. From the perspective of cost reduction and efficiency improvement, MES engineering using the modeldriven concept can be considered as the dominant method for SMEs [23]. However, little research has focused on the model-driven development of MES covering the entire engineering process, not to mention applying it to the food and beverage industry. Mizuoka and Koga [33] have introduced model-driven architecture into the development of MES for the machine processing industry with models described in XML metadata interchange, which is exported by a modeling tool from the model in UML. However, Lara et al. [34] have pointed out that the general-purpose modeling language used in model-driven engineering may not be able to fulfill the requirements from different particular application domains. Therefore, the domain-specific language should be defined and related to the specific domain. Flad et al. [35] introduced a model-driven concept for MES engineering for the food and beverage industry. It includes three steps: modeling the components in an MES solution, specifying the model information into a software-readable structure, and automatic generation of the MES solution with a generator. However, the concrete approach of these three steps to realizing the concept was not developed. Based on this, the extended MES Modeling Language (MES-ML) aiming at the automatic generation of MES has been proposed in [28]. Chen et al. [36] defined the domain-specific modeling elements as libraries for MES functions for energy consumption management and production efficiency analysis using the extended MES-ML. The introduction of the predefined modeling elements has reduced the modeling effort of the required MES solution and the programming effort of the generator. However, a completed approach covering the entire lifecycle of the MES that can be applied to the food and beverage manufacturing environment has not yet been proposed.

This paper presents an engineering approach for customizable MES using the model-driven concept. With this approach, customizing the MES is no longer tied with high programming effort but with modeling using predefined, specific modeling elements for different application domains. In addition to that, the possibility for the improvement of the generated MES solution has also been considered. Based on the use cases with real production data, the feasibility and practicality of the presented approach have been proven. In the remainder of this paper, Section 2 describes the model-driven approach in detail. Section 3 presents two use cases for typical application areas in the food and beverage industry. Section 4 evaluates the feasibility and applicability of the presented approach in Section 2. Section 5 concludes and outlines future work.

\section{A model-driven approach for engineering customizable MES}

Based on the features of food and beverage manufacturing processes and research in the area of model-driven engineering presented in Sections 1.1 and 1.2, a model-driven approach for the engineering of a customizable MES in the food and beverage industry has been developed. It consists of six phases: primary analysis, MES modeling, MES specification, MES generation, MES application, and MES improvement (Fig. 1).

To ensure barrier-free data exchange, transformation, and processing in this approach, the "Weihenstephan Standard" communication standard is introduced. The Weihenstephaner Standard is a standardized information model for communication between technical systems/production machines on the shop floor and the MES at the process control level [37]. With the Weihenstephaner Standard, the data availability from the machines and processes, as well as the processing of the data, can be consistently defined for each MES function.

\subsection{Primary analysis}

The analysis of requirements serves as the primary study before the implementation of MES. The actual state of the production plant and process, the available datasets, the required MES functions, and the demanded reports are clarified in this phase. Through the cooperation of multiple co-workers with different areas of focus and working backgrounds within the enterprise, a practicable MES that can fulfill the requirements from various viewpoints can be discovered [38].

\subsection{MES modeling}

Based on the results from the requirements analysis, the required MES is modeled in this phase. The MES is connected closely to the shop floor; moreover, the MES functions and reports, as well as components on the shop floor that provide data on the plant and production process to be processed, cannot be ignored. Therefore, the production plant, production process, MES function, and MES report should be modeled together in this phase. Figure 2 shows the metamodel of the extended MES-ML, which is able to model the MES concerning the four components and is considered suitable for automatic MES generation [28, 36]: the plant model in a tree diagram illustrating the production plants, the process model with three hierarchies describing the production 


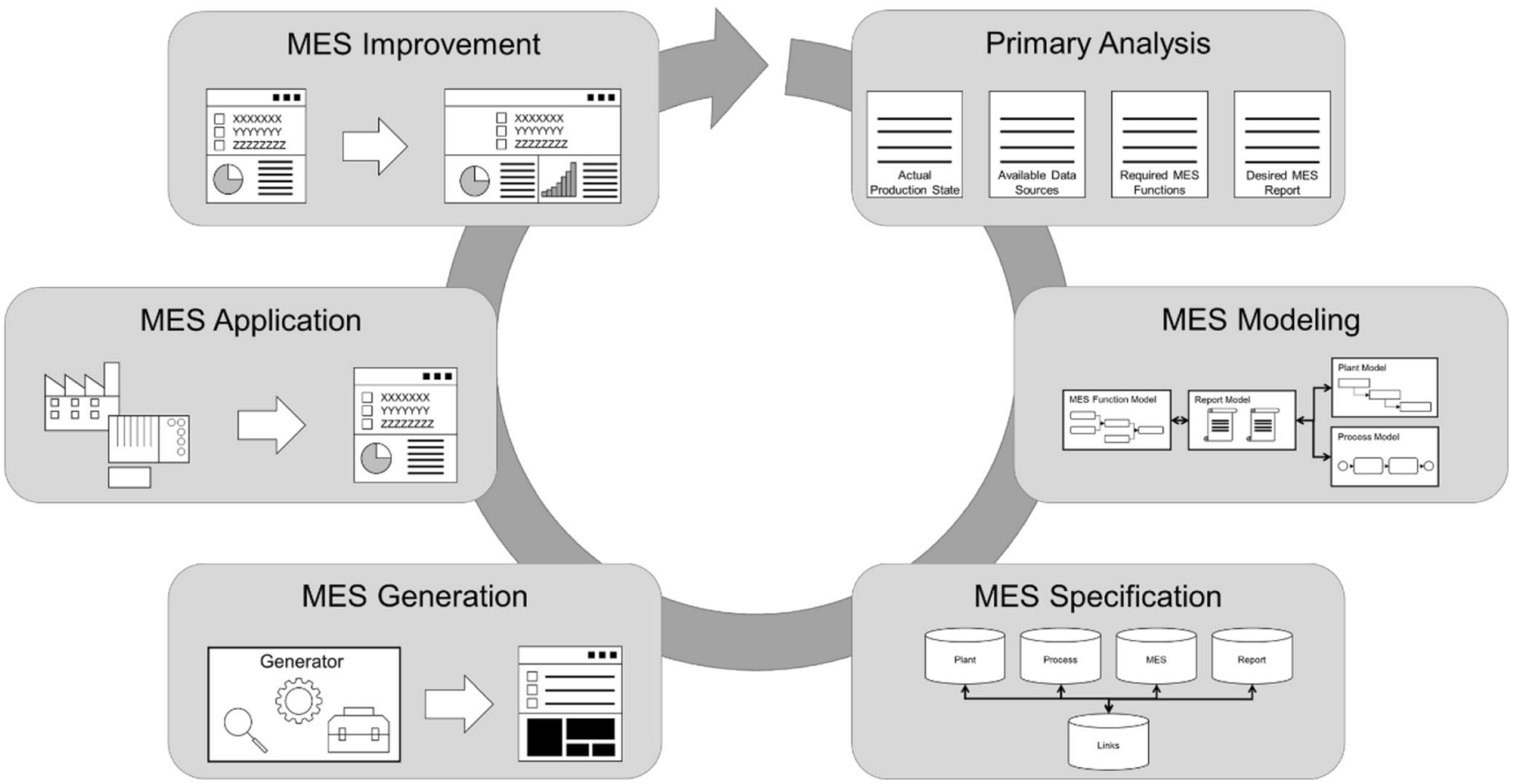

Fig. 1 Model-driven approach for engineering a customizable MES solution

processes, the MES function model representing the required MES functions composed of the predefined basic functions, and the report model linking the available data for processing and presenting the results of the MES functions.

\subsection{MES specification}

In this phase, the information in the graphical models is transformed into a software-utilizable format without losing any details. As the focus of each enterprise in terms of MES can be varied according to the manufacturing process, the MES specification must generally be designed to be usable for mixed functionalities in different implementation scenarios [39]. The database tables were chosen as the platform of the specification since it is a standard and widely used technology in the manufacturing industry, and the information in different models can be easily represented by tables with definite relationships. Figures 3, 4, and 5 present the entity-relationship model (ER model) of the tables using Chen's notation [40, 41].

Taking the two tables to specify the information of the plant model as an example, the table named "Location" has six attributes according to the hierarchy levels defined in the metamodel of the extended graphical modeling language MES-ML. With the attributes "DataPointType" and "DataPointDescription" in the table named "LocationDataPoint," detailed information about the data points can be represented. Through the attribute "location link," we can identify the affiliation between the data points and their assigned plant elements.
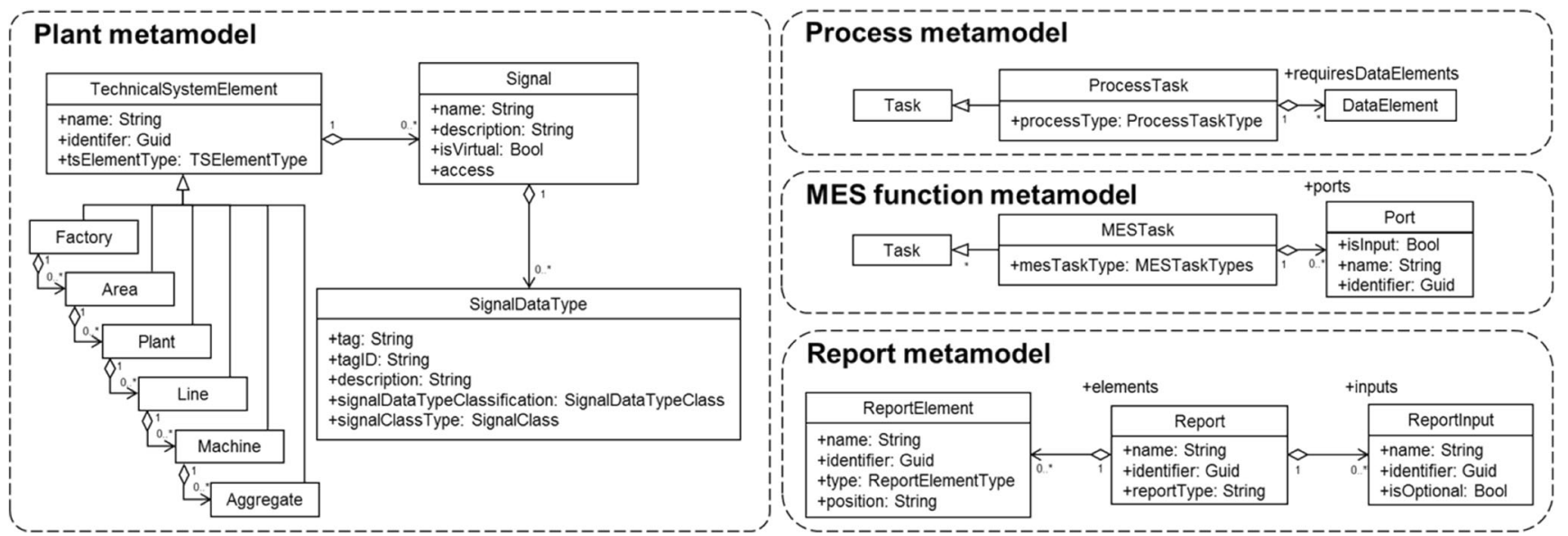

Fig. 2 Metamodel of the extended MES-ML [28, 36] 

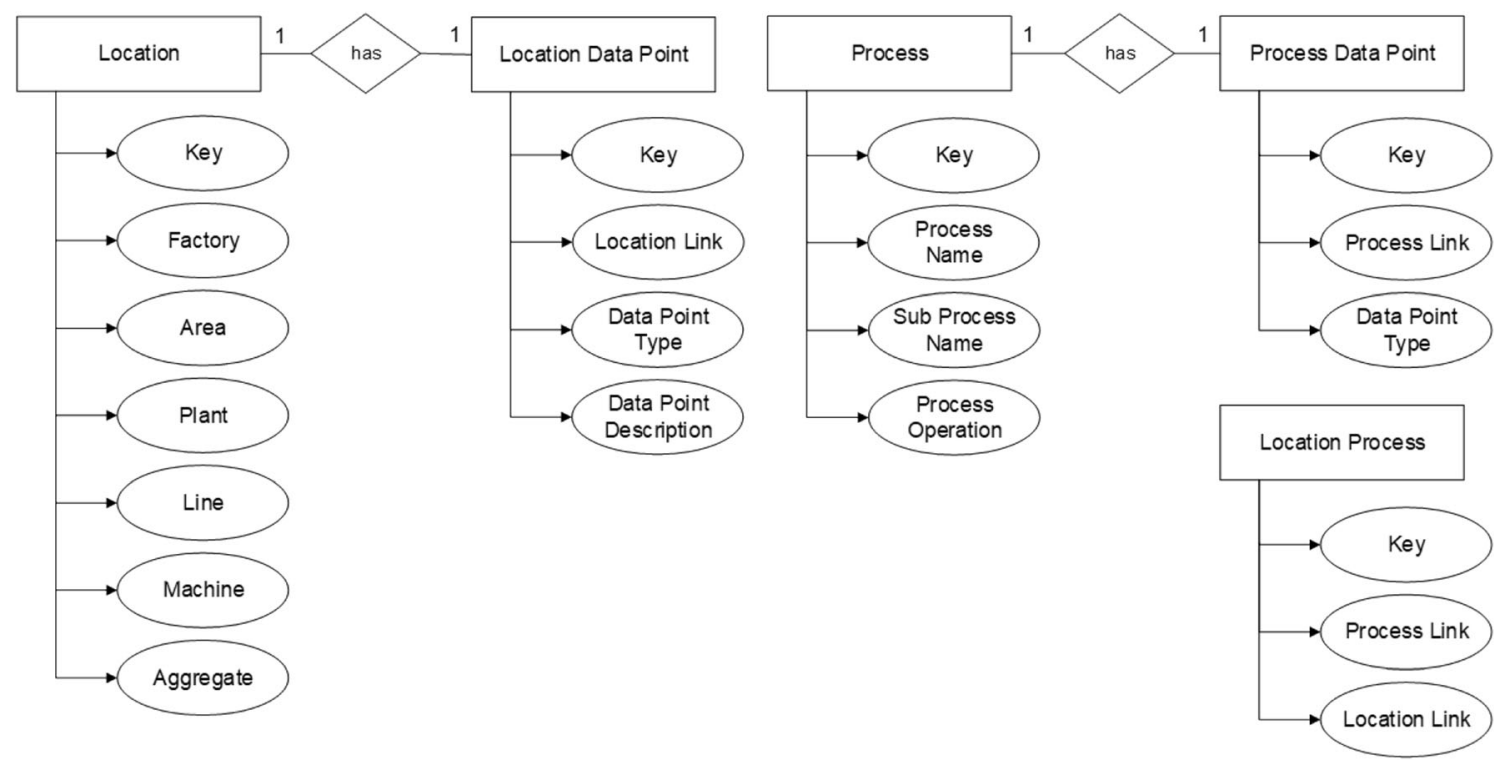

Fig. 3 ER model of the tables to represent the information in the plant model and process model

\subsection{MES generation}

In the generation phase, a user interface of the MES without specific parameters can be automatically generated by the MES generator. The generator consists of a toolbox and a connection finder. The basic functions are programmed as procedures in the toolbox, which are the fundamental elements composing different MES functions and were defined in Chen's research [36]. The use of the predefined basic functions is the precondition for automatic generation since the basic functions in the model should be known to the generator. The connection finder reads the specification that transformed from the graphical model, invokes the procedures from the toolbox, and regulates the value passing between them in the correct order modeled. Figure 6 shows the generation process in this phase.

\subsection{MES application}

The user interface of the MES produced in the generation phase was not fed with any parameters in the input area, which enable data processing by MES functions. This means that the generated MES is still generally usable and not specific to any concrete business process. In the application phase, end-users can modify the MES to fulfill their specific requirements by modifying the parameters in the input area of the user interface of the MES. After that, the MES functions behind the user interface can process the data according to the input parameters and provide the desired report in the output area.

\subsection{MES improvement}

The improvement phase is designed to deal with new requirements on the already generated MES. Since these new requirements were clarified and the related model was adopted, the MES can be improved without additional programming effort. This happens because the transformation from graphical models into a software-utilizable specification and the further generation of new MES is automatically executed. In this sense, the sustainability of the MES generated by the modeldriven approach presented can be ensured.

\section{Use cases in the processing and packaging area of the food and beverage industry}

Processing and packaging are two essential areas of operation in the food and beverage industry [13]. In the processing area, materials are mainly processed through batching and/or as continuous processes. The discrete parts manufacturing processes predominate in the packaging area. The presented approach was also applied to processing and packaging areas with two use cases to evaluate its feasibility and practicality. The first use case analyzes process-related energy consumption in the operating room of a dairy plant as the application of this approach to the processing area. In the second use case, the approach is applied to the packaging area on the bottling plant of a brewery to analyze the technical efficiency of a beer bottling line for returnable glass. According to the literature $[28,35,36]$, the prototype of an editor for modeling and transformation and a generator for automatic MES generation has been developed and used in two use cases. 


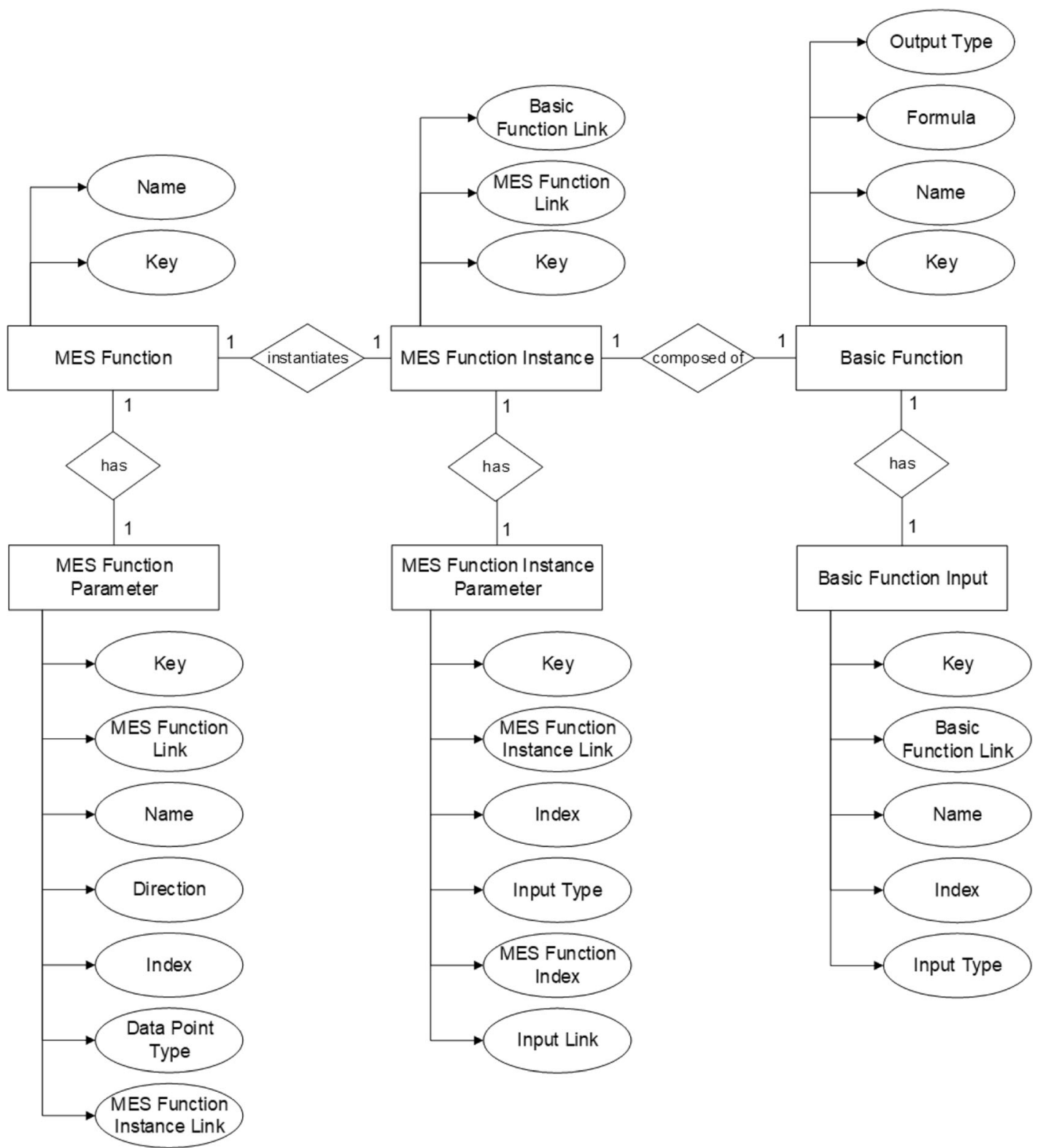

Fig. 4 ER model of the tables to represent the information in the MES function model

Fig. 5 ER model of the tables to represent the information in the report model

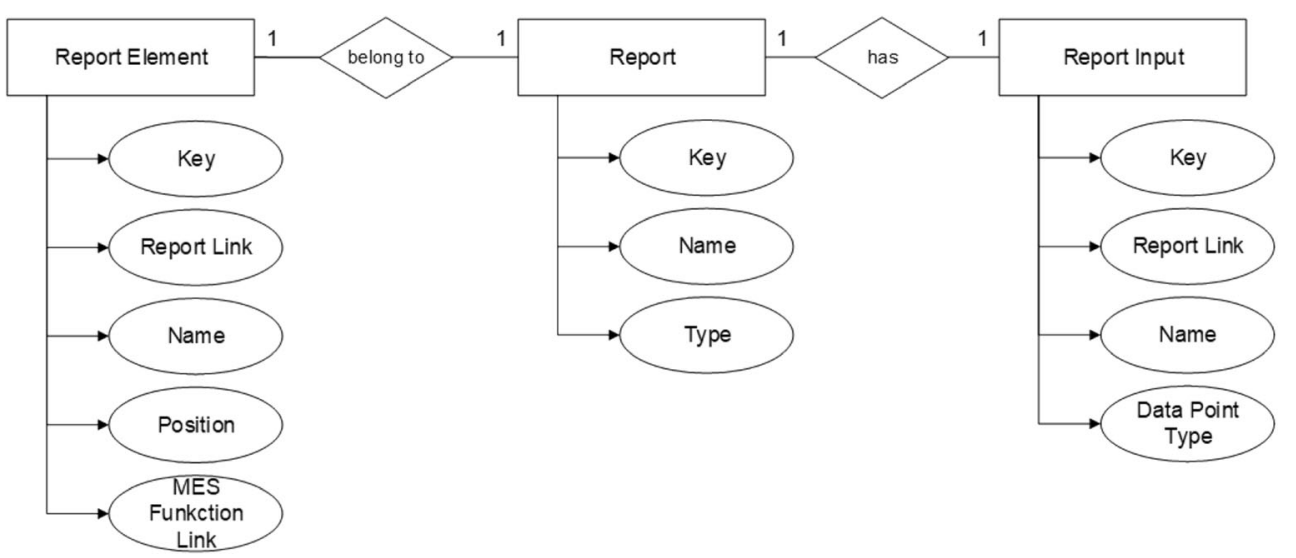




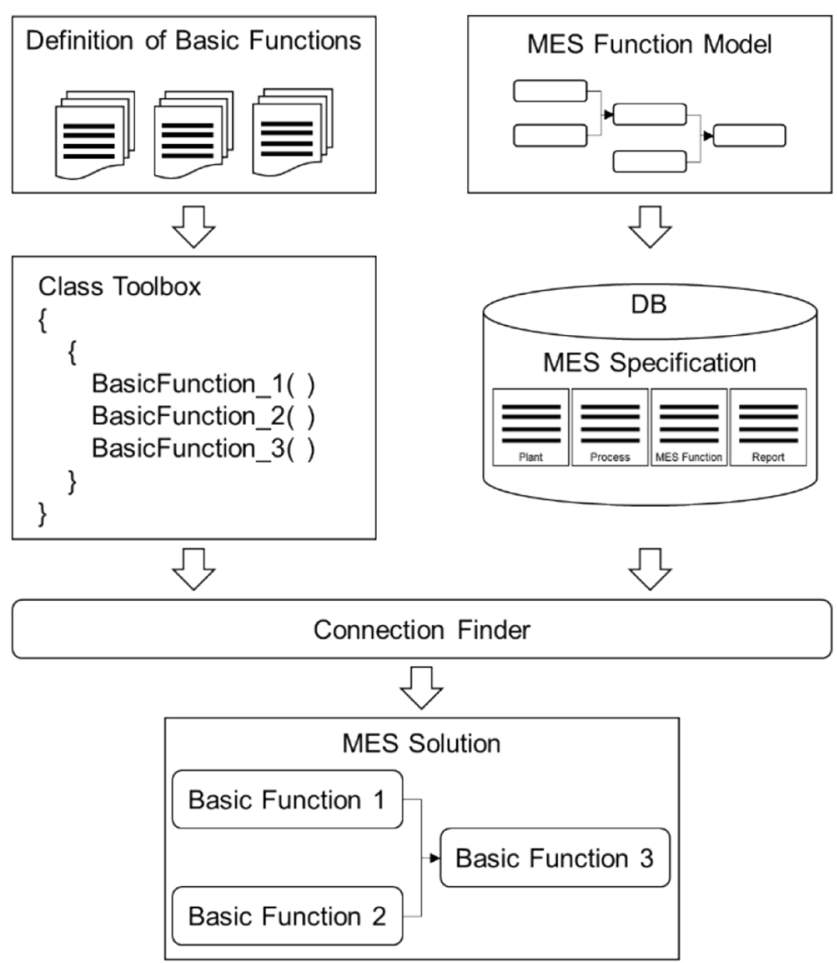

Fig. 6 The toolbox and the connection finder in the MES generator to realize the MES function

\subsection{Use case 1: processing area of a dairy}

The processing of milk is regulated by a number of laws, such as European Commission (EC) Regulation Nos. 852/2004, $853 / 2004,854 / 2004$, and 882/2004, which came into force on May 1, 2005. These laws describe the required operating processes for hygienic raw milk, heat-treated milk, and other milk-based products [42-45]. To avoid the formation of harmful germs, milk and its related products must be heated correctly and rendered compliant with the entire cold chain during processing. EC 853/2004 [43] requires that the raw milk temperature should not exceed $10^{\circ} \mathrm{C}$ on arrival at the dairy. Before pasteurization, the raw milk must be cleaned by a separator or filter. For sufficient pasteurization, a temperature/time combination of $72{ }^{\circ} \mathrm{C}$ with a contact time of $15 \mathrm{~s}$ must be achieved. In the operating room of a dairy, after the delivery of the raw milk, it is separated into skimmed milk and cream. The two components must be pasteurized and then cooled to be stored in tanks or further processed. Figure 7 shows the processing of raw milk with a centrifuge and heat exchanger in the operating room of a dairy plant.

\subsubsection{Analyzing phase of requirements and data availability}

In the operating room, the primary energy consumer is the heat exchanger, which disinfects the skimmed milk with the dosage of steam for heating and cooling the skimmed milk to be stored in tanks with refrigerant [46]. Thus, the analysis of process-related energy consumption is the main requirement in this use case. Since the machines in the operating room of the dairy are not equipped with an automated data acquisition system, the data were acquired with temperature sensors and flow meters for 1 week. Data on the milk flow rate and inlet/ outlet temperature of the heat exchanger for heating and cooling were acquired as the basis for the analysis. These data were preprocessed so that the milk flow rate and the average temperature at the inlet and outlet of the heating exchanger can be stored in a databank related to the day number with data points defined in the Weihenstephaner Standard.

\subsubsection{Modeling phase}

Plant model As presented in Fig. 7, the technical systems that attend to the processing of raw milk in the operating room are the heat exchanger and centrifuge. Figure 8 illustrates the plant model. According to the acquired data, the related data points are also assigned to the related elements in the model, namely the "WS_Vol_Flow" for the milk flow rate and the "WS_Temp_Mean" for the temperature of milk at the heat exchanger inlet and outlet.

Process model The processes in the operating room are modeled with three process hierarchy levels. The general description, "Milk Operating," is located on the process level. This process has been described at the process stage level as two parallel processes for separated cream and skimmed milk. At the level of process operation, the processing of skimmed milk is modeled in detail. The process model is presented in Fig. 9.

MES function model According to the selected day number and process name, the MES function can calculate the energy consumption related to the sterilizing and cooling process of skimmed milk. This MES function is composed of the basic functions, "Inlet Temperature Identifying," "Outlet Temperature Identifying," and mathematical operation for subtraction "Sub(x_1, x_2)" and "Multi(x_1, x_2)." Figure 10 presents the created MES function model.

The basic functions, "Inlet Temperature Identifying" and "Outlet Temperature Identifying," determine the temperature values at the inlet and outlet of the process-related heat exchanger. The difference between the two values is calculated by the basic function, "Sub(x_1, x_2)." The basic function "Multi(x_1, x_2)" has been used twice to multiply the volume flow and the constant milk heat capacity, together with the temperature difference. The value of the second basic function "Multi(x_1, x_2)" is the energy consumption of the related process that should be calculated.

Report model Since the required MES can be realized by the MES function, "Process Energy Consumption Calculation," 


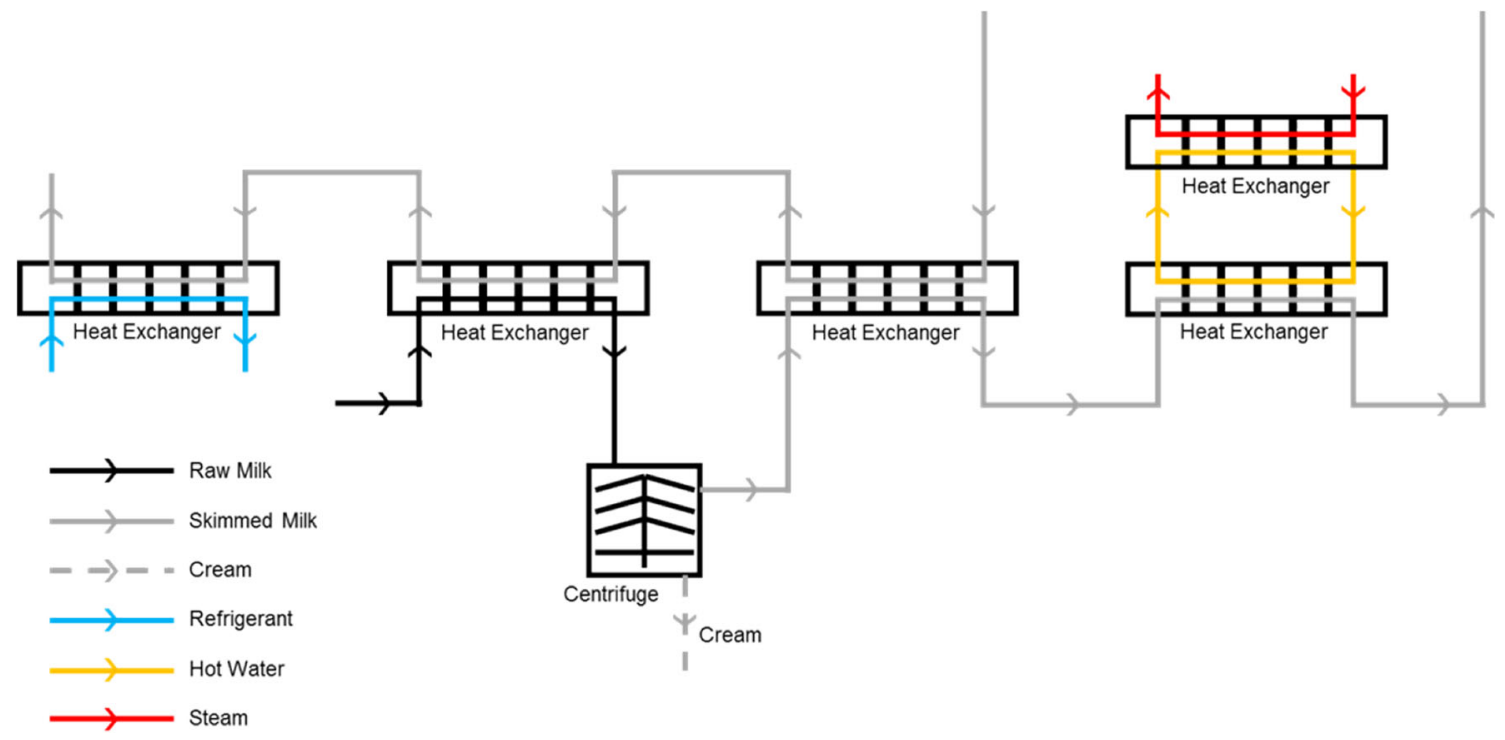

Fig. 7 Processing of raw milk in the operating room

according to the metamodel of the extended MES-ML, the report model containing the element, "Process Energy Consumption Report," presents the result of this MES function. The report model is shown in Fig. 11.

\subsubsection{Specifying phase}

With the mapping function of the prototype of the editor, the graphical information in the models was automatically transformed into database tables. As an example, the contents in the "Basic Function" and "Basic Function Input" are shown in Tables 1 and 2, representing the used basic functions and their input parameters.

\subsubsection{Generating phase and operating phase}

The basic functions were programmed as executable procedures in the toolbox of the generator. With the help of the connection finder, the sequence flow and information flow between the basic functions can be reproduced. Figure 12 presents the automatically generated graphical user interface of the generator before (left) and after (middle) the reading of the specification. The Microsoft Visual Studio 2019 serves as the developing environment for the prototype of the generator and the generator was programmed in $\mathrm{C} \#$ with WinForm.

The graphical user interface was generated in the generating phase with an input area to be parameterized for a specific application scenario. The end-user can operate the MES solution to meet their demand. Figure 12 (right) presents the report of the energy consumption of the heating process on the first day of data acquisition.

\subsubsection{Improvement of the MES}

The generated MES contains the MES function to calculate the energy consumption related to one of the selected processes. This can be improved to calculate the energy consumption of two processes simultaneously and summarize the total energy consumption. As the technical systems in the operating room and production process have not been changed, the plant model and process model remain unchanged. Figures 13 and 14 present the improved MES model and report model.

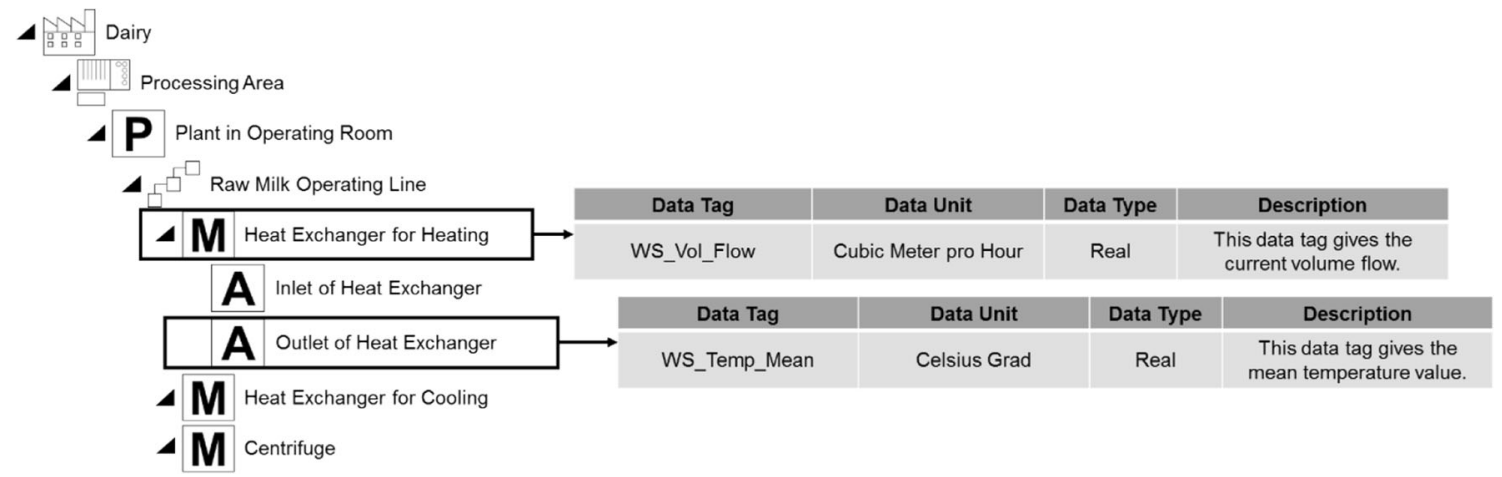

Fig. 8 Plant model of the technical systems in the operating room of a dairy plant in use case 1 
Process

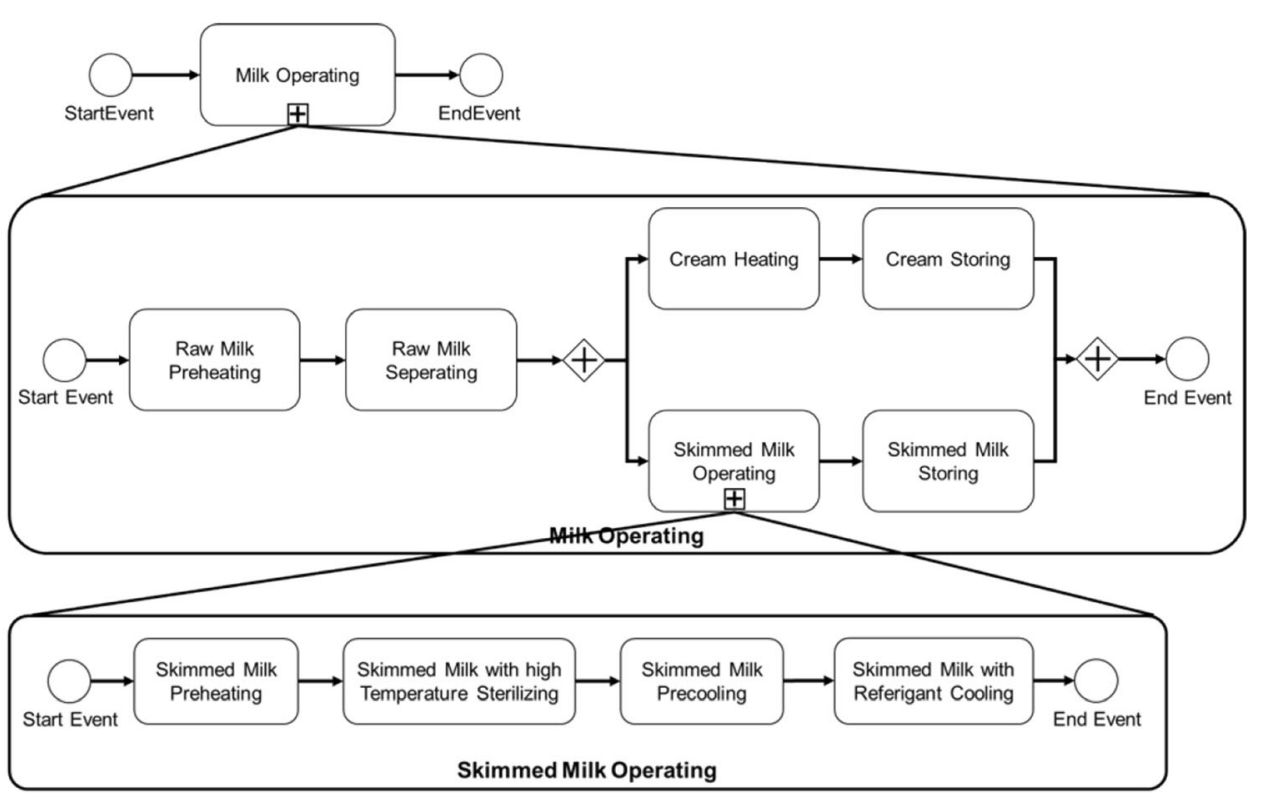

Fig. 9 Process model of milk processing in the operating room in use case 1

According to the model, new basic functions are not required to compose the improved MES function. Therefore, the toolbox of the generator must not be updated. In this sense, the new MES can be generated automatically without any additional programming effort. Figure 15 shows the generated graphical user interface of

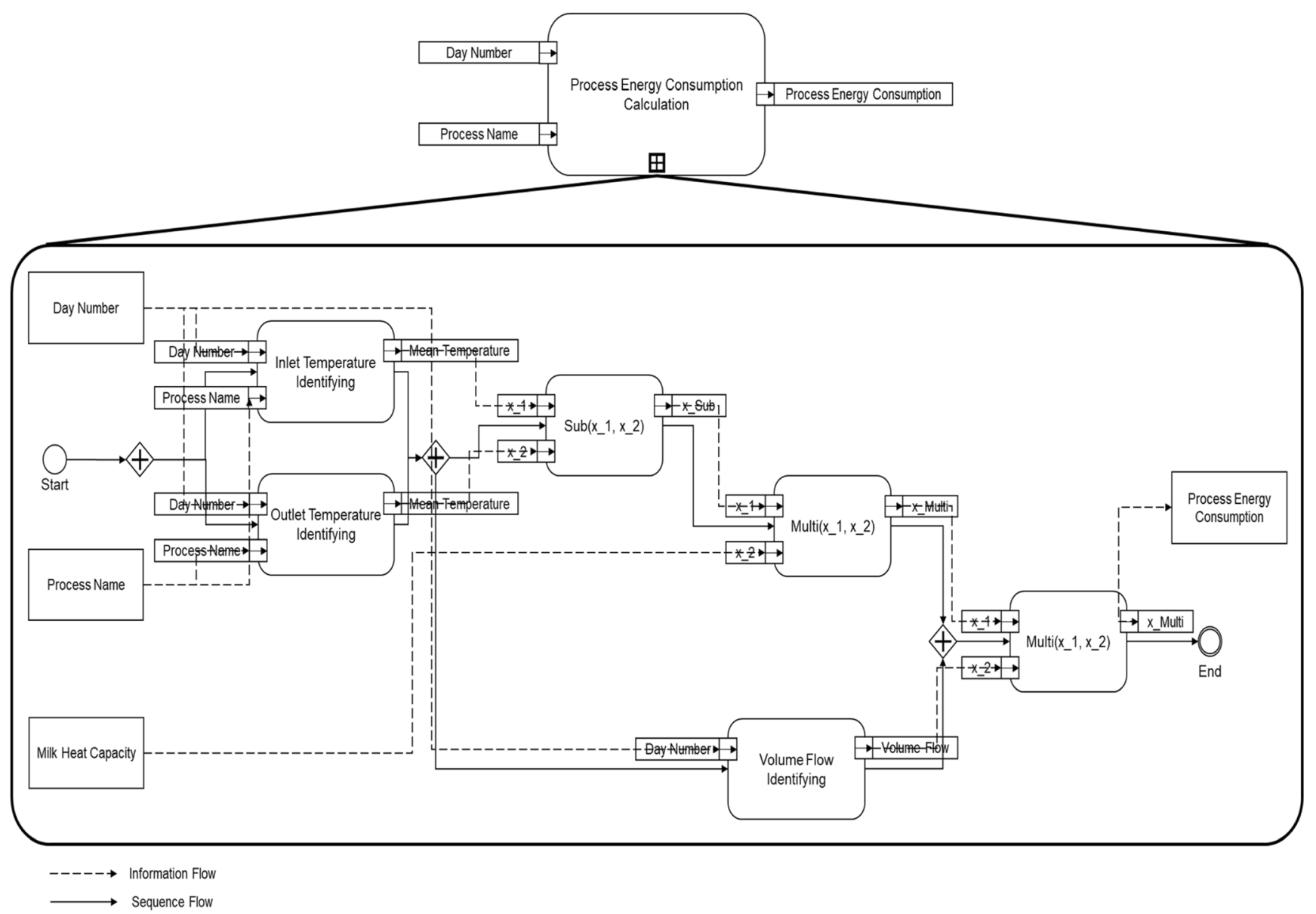

Fig. 10 MES function model to analyze process-related energy consumption in use case 1 


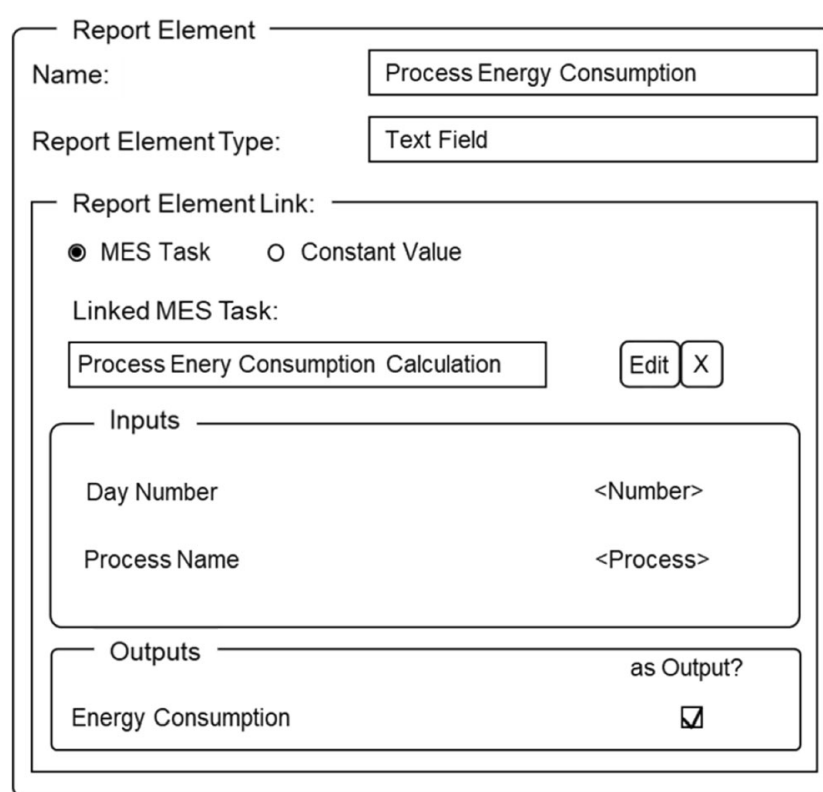

Fig. 11 Report model to indicate the process energy consumption calculated by the MES function in use case 1

the improved MES before and after specific parameterization.

\subsection{Use case 2: packaging area of a brewery}

The packaging process is the last highly automated step of modern food and beverage production. It is performed in high-performance packaging lines that involve various machines interlinked with buffering transport elements [47]. For the bottling of beer in returnable glass bottles, machines for depalletizing, unpacking, cleaning, and controlling the containers and bottles, bottle filling and capping machines, bottle labeling machines, packing palletizing and transport, are utilized [48]. The design of a typical beer bottling plant for returnable glass bottles is shown in Fig. 16.

The second use case is an industrial beer bottling and packaging plant for returnable glass bottles in a medium-sized brewery. The target plant is designed for a nominal output of 15,000 bottles/ $h$, and its main use is for filling returnable $0.33-$ 1 glass bottles. As the two phases for specifying the model information and generating the MES solution are executed automatically, only the phases for analyzing requirements,

Table 1 Specification in table "Basic Function" in use case 1

\begin{tabular}{lll}
\hline Key & Name & OutputType \\
\hline 1 & Inlet Temperature Identifying & Temperature \\
2 & Outlet Temperature Identifying & Temperature \\
3 & Sub(x_1, x_2) & x_Sub \\
4 & Multi(x_1, x_2) & x_Multi \\
\hline
\end{tabular}

Table 2 Specification in table "Basic Function Input" in use case 1

\begin{tabular}{lllll}
\hline Key & Name & BasicFunctionLink & Index & InputType \\
\hline 1 & Day Number & 1 & 0 & Number \\
2 & Process Name & 1 & 1 & Process \\
3 & Day Number & 2 & 0 & Number \\
4 & Process Name & 2 & 1 & Process \\
5 & x_1 & 3 & 0 & 1. Value \\
6 & x_2 & 3 & 1 & 2. Value \\
7 & x_1 & 4 & 0 & 1. Value \\
8 & x_2 & 4 & 1 & 2. Value \\
\hline
\end{tabular}

modeling, and application of the MES are presented in the second use case.

\subsubsection{Analyzing phase of requirements and data availability}

As mentioned in Section 1.1, to improve the production efficiency, the MES function to analyze plant production efficiency is the main focus of the packaging area in this use case. The technical efficiency $\left(\mathrm{E}_{\mathrm{S}}\right)$ defined in DIN 8743 was considered the primary efficiency indicator [49]. The bottle filling and capping machine is regarded as the central machine in the data acquisition phase since it has the lowest performance in the entire bottling line and the key influence on the quality of the final product. The data regarding the total duration of the data acquisition (with the data tag "PE_Tot_Duration"), the sellable quality output of the entire bottling line (with the data tag "WS_Good_Packages"), the set performance of the bottling and capping machine (with the data tag "WS_Set_Mach_Spd"), and the duration of its downtime (with the data tag "PE_DownTime") should be documented. The data tags with a prefix of "WS" are tags that are defined in the Weihenstephan Standard. Since there is no data tag defined in this that record the event duration, the tag prefix "PE" which stands for production efficiency was created for this use case. The data were acquired over 6 days, for $8 \mathrm{~h}$ each day. As a result, the acquired data were stored in a databank that served as the basis to be processed by the MES.

\subsubsection{MES modeling}

To analyze the production efficiency, according to DIN 8743 , no information about the process must be provided. Therefore, the process model is not created in this use case. The technical systems that participate in the beer bottling line are modeled in the plant model. According to the type of acquired data, the data points that indicate the data availability are assigned to the line level (PE_Tot_Duration, WS_Good_packages) and the bottle filling and capping machine on the machine level 

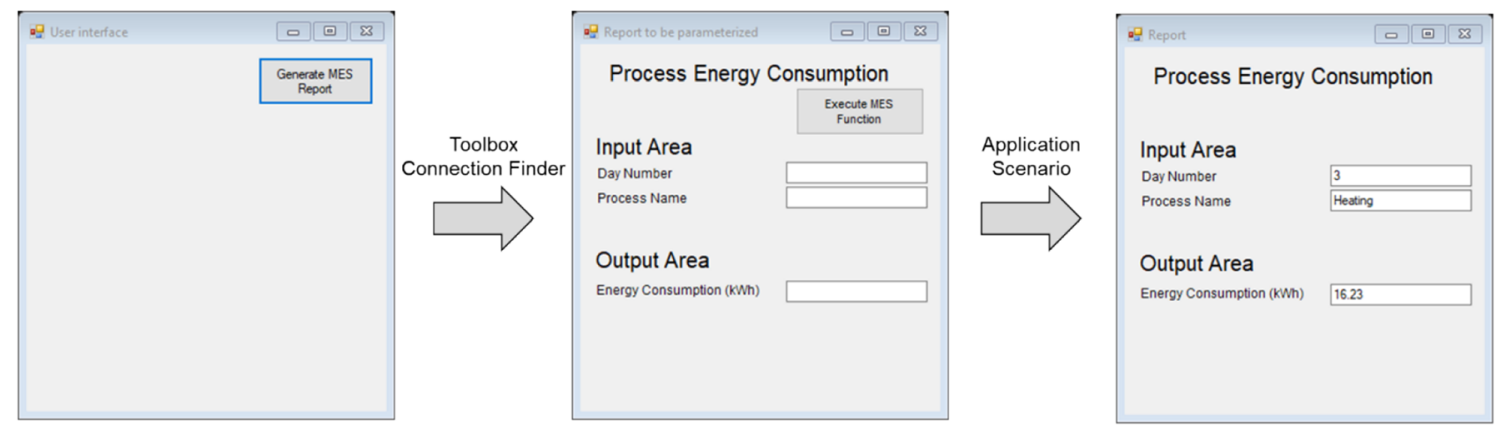

Fig. 12 Graphical user interface before (left) and after (middle) the reading of the specification and final consumption report (right) in use case 1

(WS_Set_Mach_Spd, PE_DownTime). Figure 17 shows the plant model.

The basic functions for the mathematical subtraction, multiplication, division, and calculation of the operating time, as well as the non-technical loss time, are necessary to analyze the production efficiency. Figure 18 presents the MES function model.
As defined in the metamodel of the extended MES-ML, the report model contains one report element to present the result of the MES function "Technical Efficiency Calculation" in text format. The report model includes the input parameters necessary to execute the data processing of this MES function and its result as an output parameter, and is shown in Fig. 19.

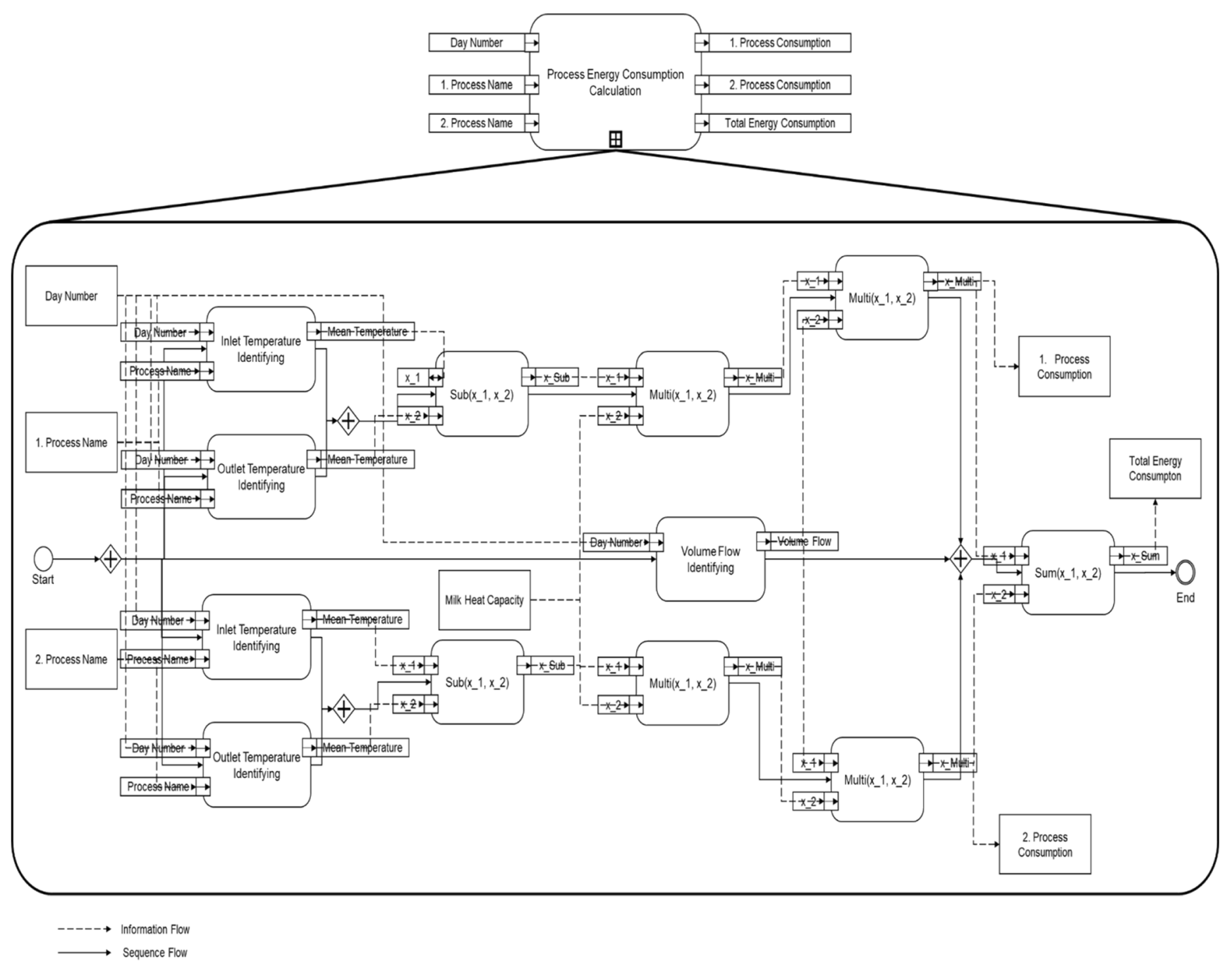

Fig. 13 Improved MES function model for use case 1 to calculate the total energy consumption of two processes 


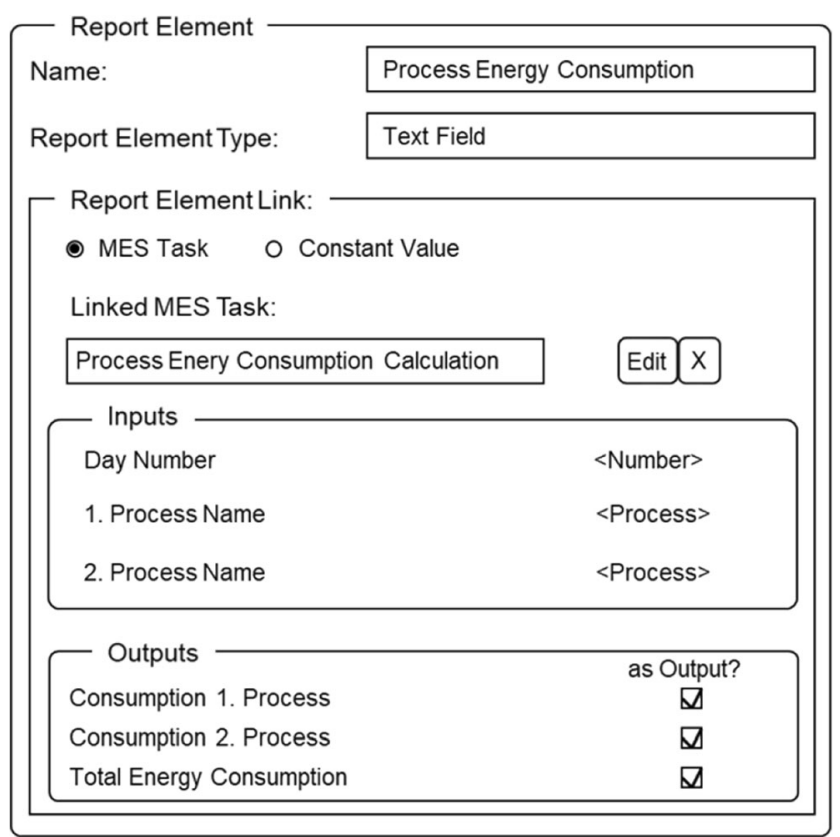

Fig. 14 Report model after the improvement of use case 1

\subsubsection{MES application}

Related to the MES function model, there is one parameter in the input area assigned by the end-user, namely the day number of the data acquisition. According to the day number, the operating time, the quality output, the not technical loss time, set performance, and the resulted technical performance are shown in the output area (Fig. 20).

\section{Evaluation}

In Section 3, the desired MES was generated successfully by using the presented approach. Based on the two use cases, the feasibility of this model-driven approach for the engineering of customizable MES has been confirmed. The procedures to realize the basic functions and routine to establish the connections of basic functions in the MES generator must be programmed in advance. On the other hand, the following steps for information transformation and MES generation were executed automatically after the model had been created. The programming and customization effort for MES engineering can be reduced through the implementation of this approach.

The standard information model, the Weihenstephaner Standard, ensures the compatibility of the information flow and data exchange during the transformation from textual requirements into a graphical model, and then into a softwarereadable specification and operational MES. However, as parts of the data points were not standardized, the compatibility of the basic functions, and from these the MES functions, may not be satisfied. Along with the further development of the Weihenstephan Standard that should contain data points covering requirements on data exchange in different domains, the data consistency and compatibility of the entire modeldriven approach can be improved.

The improvement of the MES was also considered in this approach, in which further MES functions can be integrated into the already existed MES to fulfill the new requirements. To update the MES, only the models must be modified in the modeling phase since the further phases can be executed automatically. In the first use case, the original MES was used to calculate the energy consumption related to a definite process. The MES function model and report model were expanded with more basic functions and parameters in the input and output areas, while the plant model and process model remained unchanged. After the improvement, the MES was able to calculate the energy consumption of two selected processes simultaneously and return the consumption value of each process, as well as the total energy consumption. As a result, the presented approach ensures the sustainability of the engineering process.

This model-driven approach was applied to the processing and packaging areas in the food and beverage industry, which have exact different requirements on MES: the analysis of the
Fig. 15 MES report following the improvement

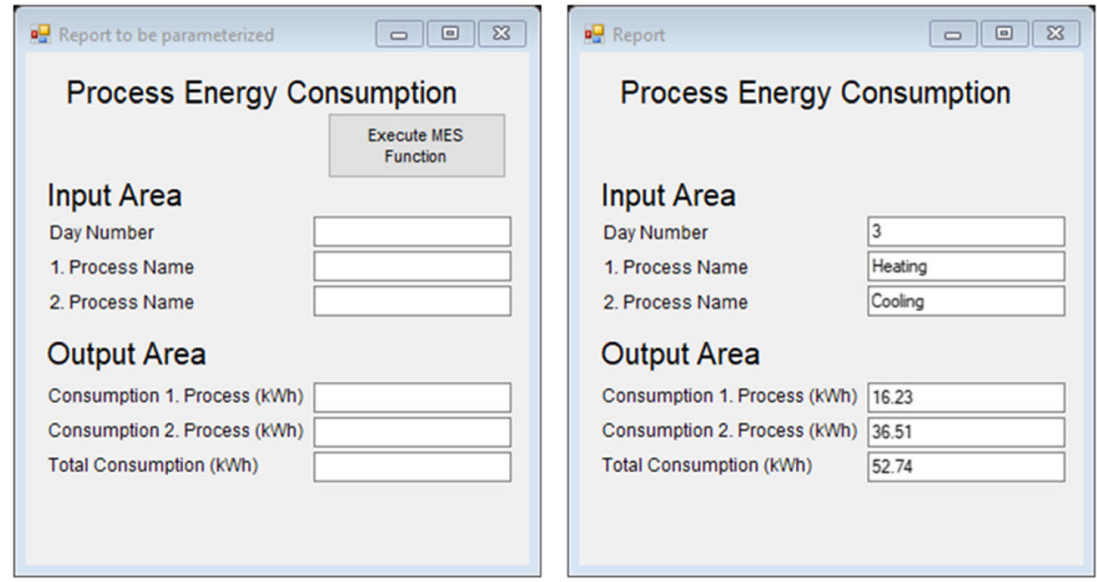


Fig. 16 Design of a beer bottling plant for returnable glass bottles

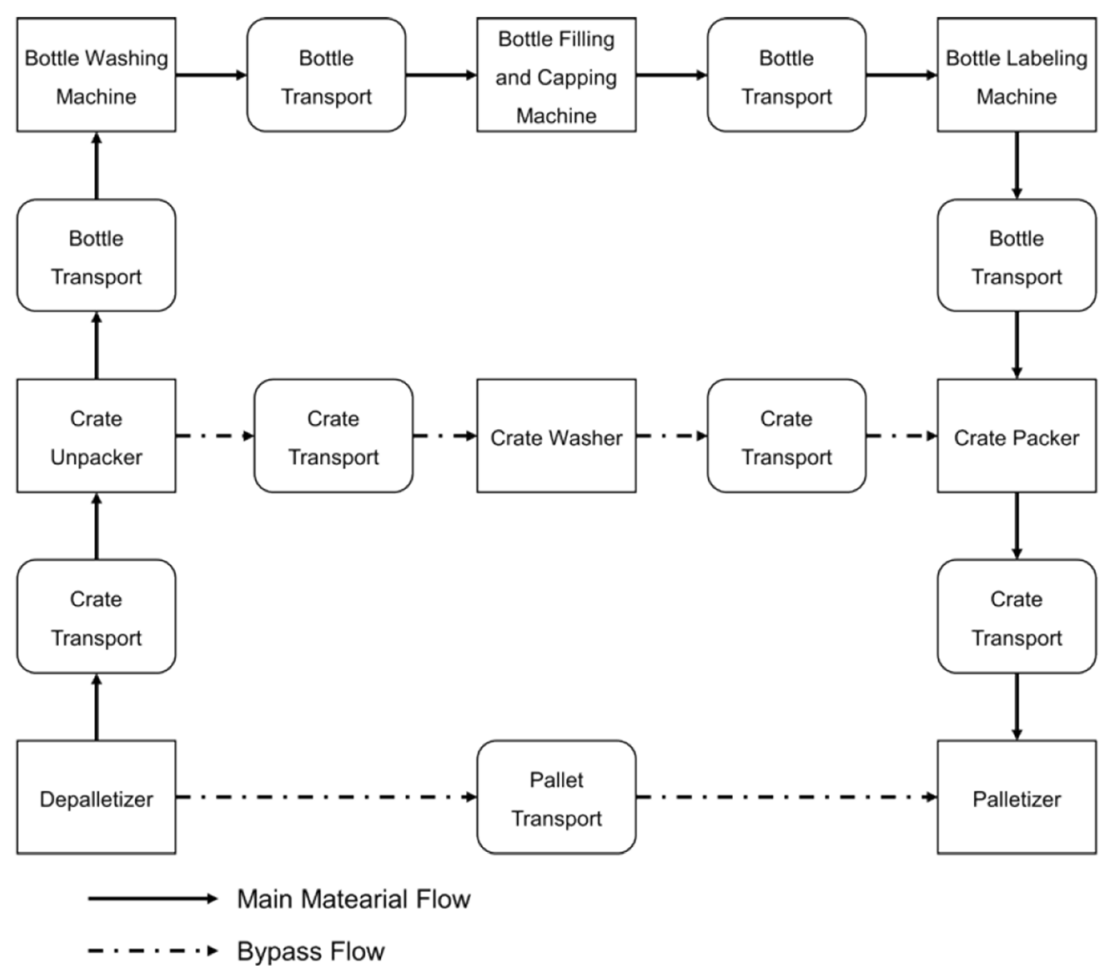

energy consumption for milk processing in the operating room of a dairy and the analysis of production efficiency for beer bottling in the filling room of a brewery. The successful application and validation with real production data have demonstrated the viability of the presented approach to the engineering of MES that requires customization. However, the engineering of an MES that can realize real-time MES functions through this approach remains to be verified since both MES are based on the processing of historical production data. The real-time functions, such as operations scheduling, product tracing, and quality management, are also the main focuses of the MES. Thus, the application of the presented approach should be extended to realize the real-time MES functions. Furthermore, more basic functions should be defined and implemented to apply this model-driven approach across a broader range, not only in the food and beverage industry but also in other industries, to evaluate the compatibility of the presented approach.

\section{Conclusions and outlook}

This paper presented a model-driven approach for engineering customizable MES in the food and beverage industry. It consists of six phases: (i) a primary analysis of the MES requirements; (ii) MES modeling with graphical modeling language of four model components; (iii) specification of the graphical information into software-readable databank tables; (iv) the

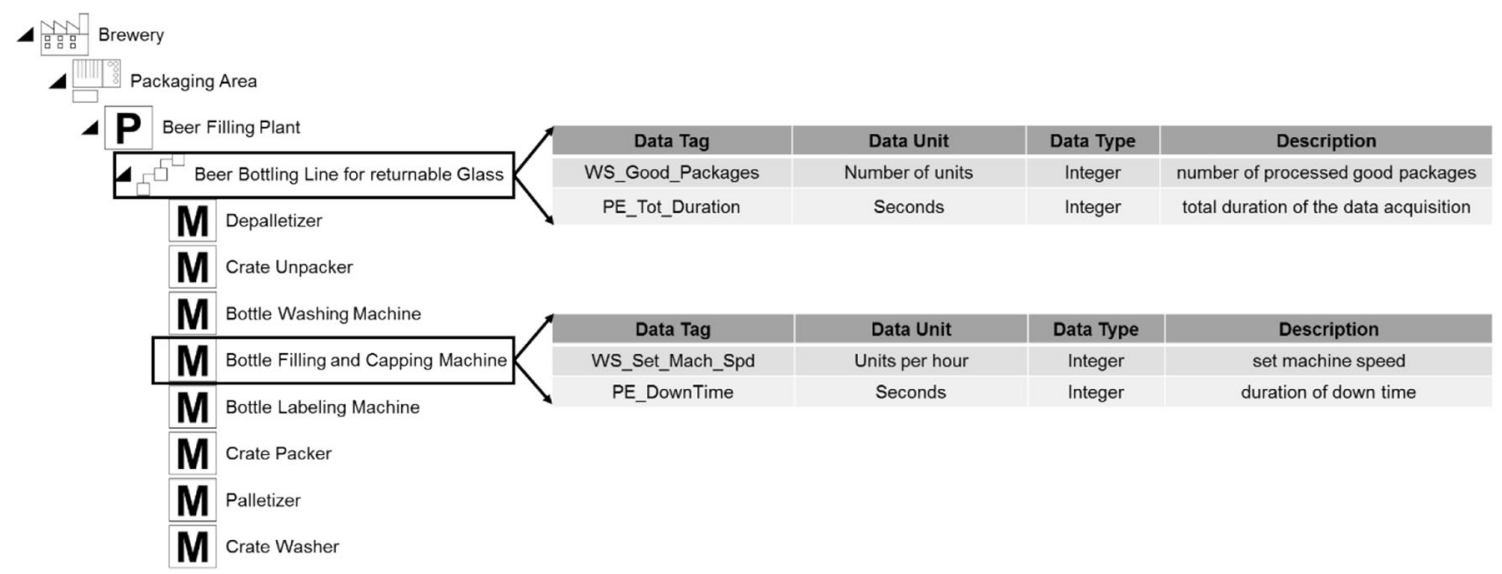

Fig. 17 Plant model of the beer bottling line for returnable glass in use case 2 


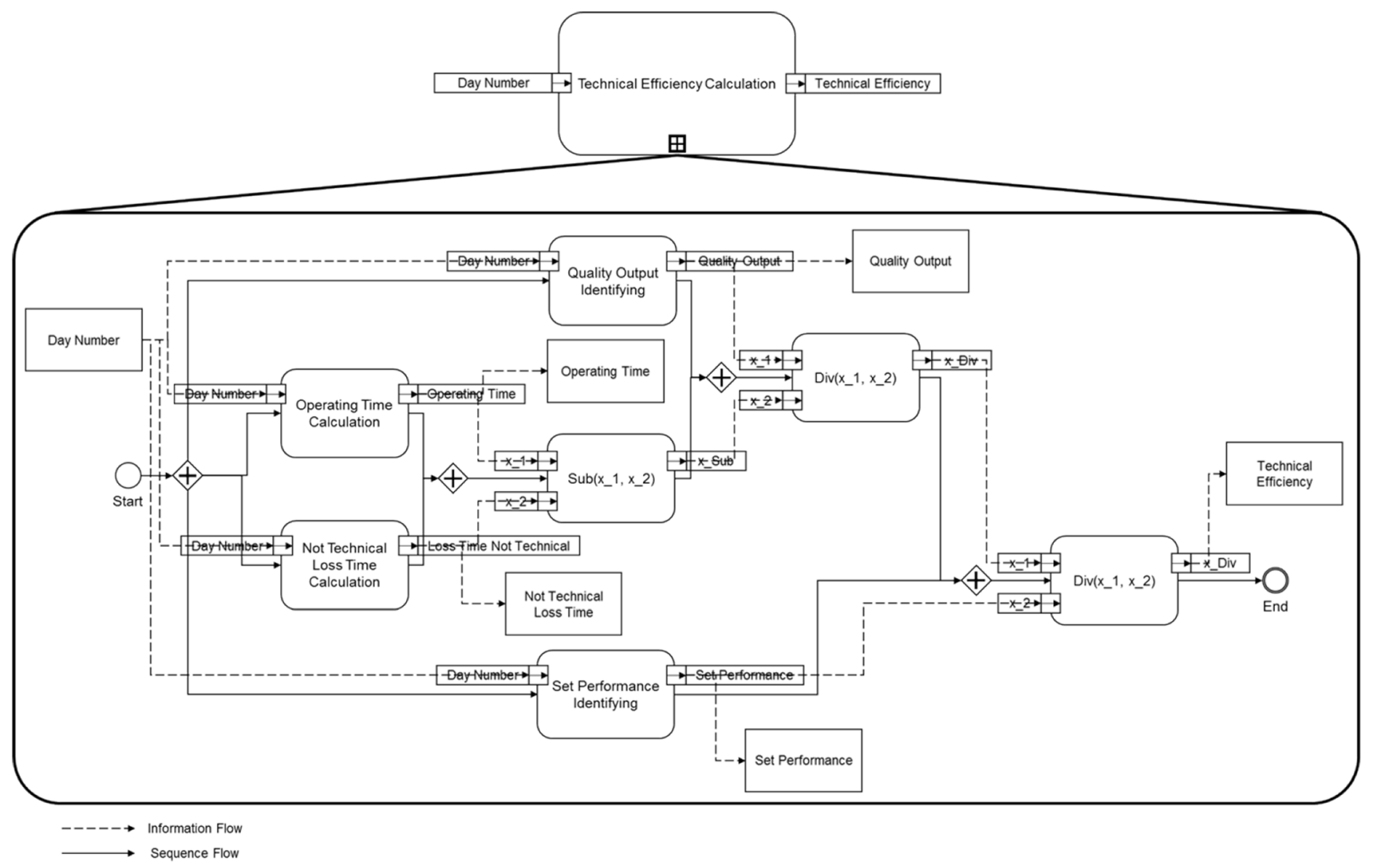

Fig. 18 MES function model to analyze the production efficiency in use case 2

automatic generation of the MES based on the specification; (v) the application of the MES to fulfill the specific scenarios; (vi) the improvement of the adopted MES in light of the new requirements. With this approach, the MES can be generated automatically after the MES has been modeled. This approach

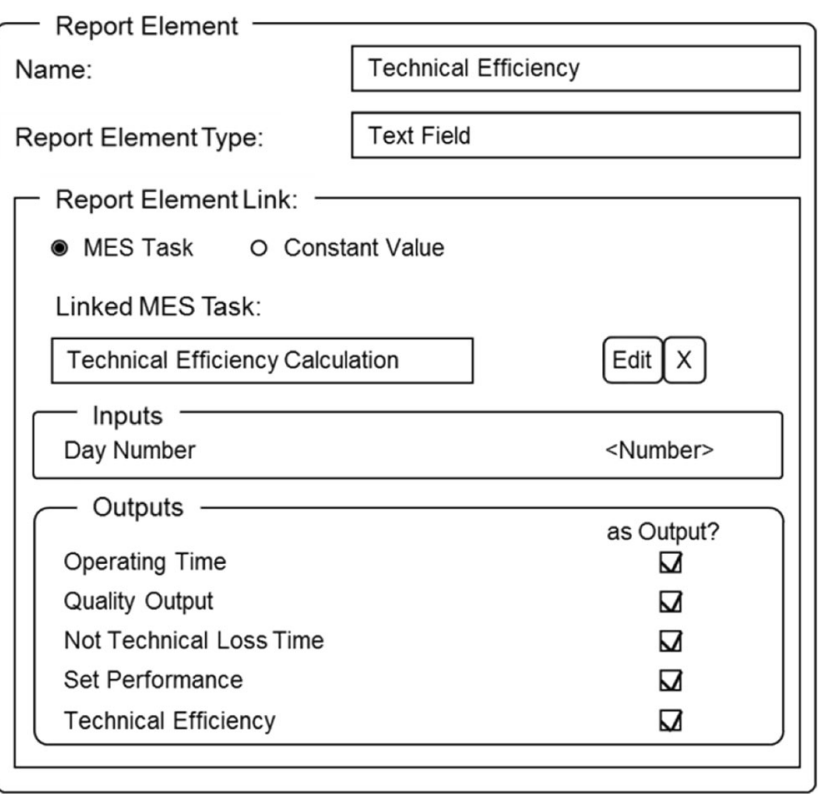

Fig. 19 Report model to analyze production efficiency in use case 2 has reduced the complexity of MES implementations with respect to programming and customization efforts. As the food and beverage manufacturers are mostly SMEs, with limited resources to invest in MES implementation, this approach is considered as a solution that promises to benefit manufacturers in this sector.

The main focus of this paper was on verifying the feasibility and practicality of the presented approach by its use cases, one from the processing area and the other from the packaging area, which are the two essential areas in the food and beverage industry. In the first use case, the MES was generated to analyze the process-related energy consumption in the operating room of a dairy. The MES in the second use case focused on the analysis of the technical efficiency of a beer bottling line in the filling room of a brewery. The requirements of the two use cases were fulfilled with the generated MES by using the presented approach. Moreover, the MES solution can be improved to react to new requirements. As a result, it has been shown that the model-driven approach presented can be used to engineer MES to fulfill different requirements, to which the generated MES should be customized.

In future work, in accordance with the results of this evaluation, collaboration with the working group of the Weihenstephan Standard is planned to define more data points corresponding to different MES functions and to achieve better integration of the Weihenstephan Standard into the model- 
Fig. 20 MES report for the production efficiency of the bottling line

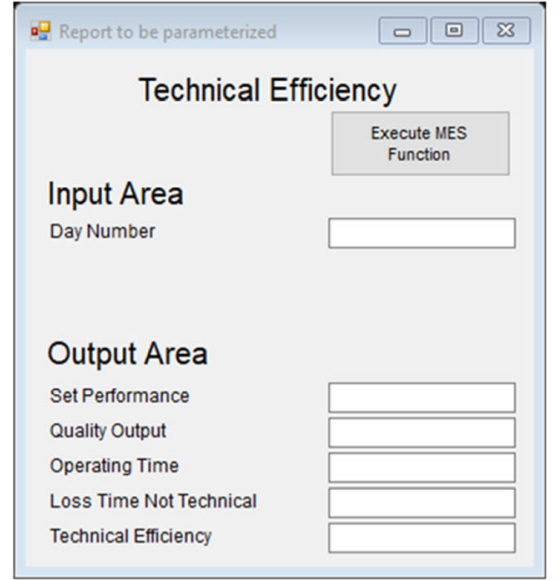

driven approach. It is also planned to analyze the requirements from other industries and define more basic functions to compose MES functions that fulfill their requirements so that the approach presented here can also be applied widely. Furthermore, essential real-time MES functions will be a focus of further development to provide the model-driven approach with higher compatibility for the generation of MES.

Code availability The code generated during the current study are available from the corresponding author on reasonable request.

Author contribution Xinyu Chen: conceptualization, methodology, software, data curation, writing - original draft, writing - review \& editing; Christoph Nophut: methodology, writing-review \& editing; Tobias Voigt: writing - review \& editing, supervision. All authors have read and agreed to the published version of the manuscript

Funding Open Access funding enabled and organized by Projekt DEAL.

Data availability The datasets used and/or analyzed during the current study are available from the corresponding author on reasonable request.

\section{Declarations}

Ethics approval Not applicable.

\section{Consent to participate Not applicable.}

Consent to publish Not applicable.

Conflict of interest The authors declare no competing interests.

Open Access This article is licensed under a Creative Commons Attribution 4.0 International License, which permits use, sharing, adaptation, distribution and reproduction in any medium or format, as long as you give appropriate credit to the original author(s) and the source, provide a link to the Creative Commons licence, and indicate if changes were made. The images or other third party material in this article are included in the article's Creative Commons licence, unless indicated otherwise in a credit line to the material. If material is not included in the article's Creative Commons licence and your intended use is not permitted by statutory regulation or exceeds the permitted use, you will need to obtain permission directly from the copyright holder. To view a copy of this licence, visit http://creativecommons.org/licenses/by/4.0/.

\section{References}

1. MESA, International White Paper: MES explained: a high level vision, 1997

2. International Electrotechnical Commision, IEC 62264-1 Enterprise-control system integration - Part 1: Models and terminology, 2013

3. Verein Deutscher Ingenieure, VDI 5600 - Part 1: Manufacturing Execution Systems (MES), Beuth Verlag GmbH, Berlin, 2016

4. Rösch S, XSchütz S, Weißenberger B, Chen X, Voigt T, B. VogelHeuser (Eds.), Durchgängiges MES-Engineering als Grundlage für Industrie 4.0, 2016.

5. FoodDrinkEurope, data \& trends - EU Food and Drink Industry 2019, 2019.

6. Monforti-Ferrario F, Pascua IP, Motola V, Banja M, Scarlat N, Medarac H, Castellazzi L, Labanca N, Bertoldi P, Pennington D (2015) Energy use in the EU food sector: State of play and opportunities for improvement. Publications Office

7. Mukherjee K (2008) Energy use efficiency in US manufacturing: a nonparametric analysis. Energy Econ 30:76-96

8. United Nations (1997) United Nations framework convention on climate change, Kyoto Protocol. Kyoto 19:497

9. UNFCCC (2009) Report of the Conference of the Parties on its fifteenth session: Part Two: Action taken by the Conference of the Parties at its fifteenth session. Decisions adopted by the Conference of the Parties, Copenhagen

10. F. Jovane, H. Yoshikawa, L. Alting, C.R. Boer, E. Westkamper, D. Williams, M. Tseng, G. Seliger, AM Paci, The incoming global technological and industrial revolution towards competitive sustainable manufacturing, CIRP Ann 57 (2008) 641-659.

11. Maxime D, Marcotte M, Arcand Y (2006) Development of ecoefficiency indicators for the Canadian food and beverage industry. $\mathrm{J}$ Clean Prod 14:636-648

12. Bunse K, Vodicka M, Schönsleben P, Brülhart M, Ernst FO (2011) Integrating energy efficiency performance in production management-gap analysis between industrial needs and scientific literature. J Clean Prod 19:667-679

13. N.P. Mahalik, A.N. Nambiar, Trends in food packaging and manufacturing systems and technology, trends in food science \& technology 21 (2010) 117-128. 
14. R. Weinekötter, Compact and efficient continuous mixing processes for production of food and pharmaceutical powders, trends in food science \& technology 20 (2009) S48-S50.

15. Borges Lopes R, Freitas F, Sousa I (2015) Application of lean manufacturing tools in the food and beverage industries. $\mathrm{J}$ Technol Manag Innov 10:120-130

16. Desai DA, Kotadiya P, Makwana N, Patel S (2015) Curbing variations in packaging process through Six Sigma way in a large-scale food-processing industry. Journal of Industrial Engineering International 11:119-129

17. Cottyn J, van Landeghem H, Stockman K, Derammelaere S (2011) A method to align a manufacturing execution system with Lean objectives. Int J Prod Res 49:4397-4413

18. Palanisamy S, Siddiqui S (2013) Changeover time reduction and productivity improvement by integrating conventional SMED method with implementation of MES for better production planning and control. International Journal of Innovative Research in Science, Engineering and Technology 2:7961-7974

19. Vogel-Heuser B, Kegel G, Wucherer K (2009) Global information architecture for industrial automation. atp magazin 51:108-115

20. M. Brettel, N. Friesderichsen, M. Keller, M. Rosenberg, How virtualization, decentralization and network building change the manufacturing landscape: an industry 4.0 perspective, FormaMente 12 (2017).

21. E. Arica, D.J. Powell, Status and future of manufacturing execution systems, in: 2017 IEEE International Conference on Industrial Engineering and Engineering Management (IEEM), pp. 20002004.

22. Chen X, Voigt T (2020) Implementation of the Manufacturing Execution System in the food and beverage industry. J Food Eng 278:109932

23. Bateman RJ, Cheng K (2006) Extending the product portfolio with 'devolved manufacturing': methodology and case studies. Int J Prod Res 44:3325-3343

24. Bär R, Voigt T (2017) WS Brew - Weihenstephaner Standards for the brewing process. Freising, Germany

25. Meyers S, Schmitt B, Chester-Jones M, Sturm B (2016) Energy efficiency, carbon emissions, and measures towards their improvement in the food and beverage sector for six European countries. Energy 104:266-283

26. L. Fei, Manufacturing execution system design and implementation, in: 2010 2nd International Conference on Computer Engineering and Technology, 559 - 562.

27. G. Gutermuth, B. Schroeter, R. Drath, N. Li, C. Messinger, A novel approach to measure engineering efficiency of automation projects, in: Proceedings of the 2014 IEEE Emerging Technology and Factory Automation (ETFA), IEEE, pp. 1-8.

28. B. Weißenberger, S. Flad, X. Chen, S. Rösch, T. Voigt, B. VogelHeuser, Model driven engineering of manufacturing execution systems using a formal specification, in: 2015 IEEE 20th Conference on Emerging Technologies \& Factory Automation (ETFA), IEEE, pp. $1-8$.

29. Harel D, Rumpe B (2004) Meaningful modeling: what's the semantics of "semantics"? Computer 37:64-72

30. R. France, B. Rumpe, Model-driven development of complex software: a research roadmap, in: Future of Software Engineering (FOSE'07), IEEE, pp. 37-54.

31. J.O. Ringert, A. Roth, B. Rumpe, A. Wortmann, Code generator composition for model-driven engineering of robotics component \& connector systems, arXiv preprint arXiv:1505.00904 (2015).

32. J. Hutchinson, M. Rouncefield, J. Whittle, Model-driven engineering practices in industry, in: Proceedings of the 33rd International Conference on Software Engineering, pp. 633-642.
33. K. Mizuoka, M. Koga, MDA development of Manufacturing Execution System based on automatic code generation, in: Proceedings of SICE Annual Conference 2010, IEEE, pp. 31033106

34. de Lara J, Guerra E, Cuadrado JS (2015) Model-driven engineering with domain-specific meta-modelling languages. Softw Syst Model $14: 429-459$

35. S. Flad, B. Weißenberger, X. Chen, S. Rösch, T. Voigt, Automatische Generierung von Fertigungs-Managementsystemen, in: Handbuch Industrie 4.0 Bd. 2, Springer, 2017, pp. 349-368.

36. Chen X, Gemein F, Flad S, Voigt T (2018) Basis for the modeldriven engineering of manufacturing execution systems: modeling elements in the domain of beer brewing. Comput Ind 101:127-137

37. A. Kather, T. Voigt, Weihenstephan Standards for the production data acquisition in bottling plants: Part 1: Physical Interface Specification Part 2: Content Specification of the Interface Part 3: Data Evaluation and Reporting Part 4: Inspection and Safe Operation, TUM, Lehrstuhl für Lebensmittelverpackungstechnik (2010).

38. Witsch M, Vogel-Heuser B (2012) Towards a formal specification framework for manufacturing execution systems. IEEE Transactions on Industrial Informatics 8:311-320

39. Saenz de Ugarte B, Artiba A, Pellerin R (2009) Manufacturing execution system-a literature review. Prod Plan Control 20:525539

40. Chen PP-S (1976) The entity-relationship model - toward a unified view of data. ACM transactions on database systems (TODS) 1:9 36

41. P. Chen, Entity-relationship modeling: historical events, future trends, and lessons learned, in: Software pioneers, Springer, 2002, pp. 296-310.

42. European Commission, Regulation (EC) No 852/2004 of the European Parliament and of the Council of 29 April 2004 on the hygiene of foodstuffs, 2004.

43. European Commission, Regulation (EC) No 853/2004 of the European Parliament and of the Council of 29 April 2004 laying down specific rules for food of animal origin, 2004.

44. European Commission, Regulation (EC) No 854/2004 of the European Parliament and of the Council of 29 April 2004 laying down specific rules for the organisation of official controls on products of animal origin intended for human consumption, 2004.

45. European Commission, Regulation (EC) No 882/2004 of the European parliament and of the council of 29 April 2004 on official controls performed to ensure the verification of compliance with feed and food law, animal health and animal welfare rules, 2004.

46. T. Huppertz, A.L. Kelly, A.Y. Tamime, Milk processing and quality management, Wiley-Blackwell Pub./Society of Dairy Technology, 2009.

47. Osterroth I, Klein S, Nophut C, Voigt T (2017) Operational state related modelling and simulation of the electrical power demand of beverage bottling plants. J Clean Prod 162:587-600

48. Ir. G.J.A.P. Nollen, Performance measurement methods for root cause detection in packaging lines - a case study on beer bottling. Thesis for master Management, Nederland, 2018.

49. Deutsches Institut für Normung, Packaging machines and packaging lines - key figures to characterise operation behaviour and requirements for data collection in an acceptance test, Beuth Verlag GmbH, Berlin, 2014.

Publisher's note Springer Nature remains neutral with regard to jurisdictional claims in published maps and institutional affiliations. 